\title{
Two Handed Mid-Air Gestural HCI: Point + Command
}

\author{
Matthias Schwaller, Simon Brunner, and Denis Lalanne \\ Department of Informatics, University of Fribourg, 1700 Fribourg, Switzerland \\ firstname. lastname@unifr.ch, \\ brunner.simon@gmail.com
}

\begin{abstract}
This paper presents work aimed at developing and evaluating various two-handed mid-air gestures to operate a computer accurately and with little effort. The main idea driving the design of these gestures is that one hand is used for pointing, and the other hand for four standard commands: selection, drag \& drop, rotation and zoom. Two chosen gesture vocabularies are compared in a user evaluation. The paper further presents a novel evaluation methodology and the application developed to evaluate the four commands first separately and then together. In our user evaluation, we found significant differences for the rotation and zooming gestures. The iconic gesture vocabulary had better performance and was better rated by the users than the technological gesture vocabulary.
\end{abstract}

Keywords: Gestural interfaces, Two-hand interaction, User evaluation.

\section{Introduction}

Gestural user interfaces have become omnipresent in recent years. At the time of writing, the Microsoft Kinect device is one of the most used devices to recognize hands-free gestures. The great success of the Kinect device is mostly due to its low price and its accuracy for body tracking. The device comes with an RGB camera and a depth sensor, both with a resolution of about $640 * 480$ pixels and with a frame rate of $30 \mathrm{fps}$.

Until now, most hands-free gestures required big arm movements to recognize them. This paper aims at studying subtle two handed gestures in order to perform basic gestural interaction to operate standard programs on a personal computer. We decided to use a two handed paradigm with one hand for pointing and the second hand for the gestural interaction because (1) most of the users in Nancel et al. [1] rated two handed gestures as less tiring than one handed gestures, (2) there are more possibilities for different commands and (3) the gesture cannot influence the pointing accuracy (for instance a selection) and it further allows moving the pointer while a command is done.

Two different sets of gestures have been designed and compared through a user evaluation. Since there is no standard methodology to compare gestures, other than for a selection of gestures, we compared the 3 other gestures with small repetitive tasks consisting of manipulating geometrical objects. Both gesture vocabularies are 
composed of four commands: selection, drag \& drop, rotation and zoom. The drag \& drop command is composed of a selection and a release. The first gesture vocabulary is technology driven, meaning that this vocabulary is designed to guarantee robust gesture recognition, while the second gesture vocabulary is user centered and is designed to have easy-to-use and easy to remember gestures.

The presented evaluation application permits to evaluate the four commands (selection, drag \& drop, rotate and zoom) first separately and then together. The selection command evaluation is based on part 9 of the ISO 9241 standard for non-keyboard input devices and Fitt's law (multi-directional tapping test). For the other commands geometric objects have to be moved, rotated and/or resized. This gesture evaluation application is another contribution of this paper.

The remainder of the paper is structured as follows: First, we give an overview of some related work and then present the two gesture vocabularies. Furthermore, we present the test application and the evaluation with its various results. Finally we discuss the results, articulate a conclusion and discuss future work.

\section{Related Work}

Various research work has been done in the past concerning the recognition of two handed gesture input. Nancel et al. [1] presented an evaluation where they compared uni- vs. bimanual interaction. In their study they found that two handed techniques where less tiring than one handed techniques. They used the same paradigm as we do, namely to use the right hand for pointing and the left hand for gestural interactions. In their study they found that 3D in the air gestures are more tiring than gestures with either a 2D surface or a 1D path. Therefore we created more subtle gestures which require less movement and should therefore be less tiring. In their paper they also presented an interesting approach to evaluate moving and zooming of virtual circles. We use a similar approach to evaluate the zoom and the rotate gestures.

A Kinect as a sensor for two handed interaction was also used by Gallo et al. [2]. They used the same selection gesture as we use in the iconic vocabulary. For the rotation and the zooming they used both hands. We, on the contrary, use only one hand for commands and the other hand for pointing to the object we would like to modify.

A bimanual handle bar metaphor was introduced by Song et al. [3]. In their implementation they used two pointing gestures to position a handle bar, which is in fact a virtual stick that traverses the virtual object. Further, to manipulate the objects two fists were used to stretch or rotate this handle bar which zoomed or rotated the objects. Since we use only one hand for the commands and our gestures need less movement, our gestures therefore need less effort.

Ziegelbaum et al. [4] did some important work on two handed interaction. Their work was built on the g-speak by Oblong ${ }^{1}$. For their rotation and translation they used two pinch gestures (thumb and index finger touching). The movement which the user needs to do to generate commands is bigger than in our work. Furthermore, for their system the user has to stand, while for our system the user can sit in a chair with armrests.

\footnotetext{
${ }^{1}$ http: / / www . oblong.com/
} 
The WUW which is also known as Sixthsense was introduced by Mistry et al. [5] and proposed two handed zoom in and zoom out gestures very similar to the zoom gesture presented by Ziegelbaum et al. [4]. Their system is wearable and therefore the user has to wear a webcam on his chest.

The two handed zoom and rotation gestures presented by Kamel Boulos et al. [6]. are used for navigating in Google Earth. To navigate the two hands have to make fists the use is then very similar to using Google Earth with a touch screen. Instead of touching the screen with one finger, the user has to make a fist and can thus zoom and rotate with two fists. Other pan and zoom gestures to navigate in Google Earth were presented by Stellmach et al. [7]. In their study they compared 4 pan and zoom alternatives. They found that the gestures, in which the non-dominant hand had to be held up while performing the gestures, were faster than two handed gestures where both hands interacted for zooming, although the user commented that it was painful to constantly hold up their hand. For our gestures, the gestural command is done with the left hand, while the right hand is pointing to the object which has to be manipulated. This gesture is therefore somehow close to their gesture with the difference that our right hand does not have to be help up over the shoulder but is pointing in a more comfortable position.

Another two handed scenario was introduced by Gustafson et al. [8] and called "Imaginary Interfaces". In their approach they used the left hand to show in the air where the user wants to draw and the right hand was used for drawing. They have a similar gesture paradigm because in their system, as in ours, the right hand is used for pointing / drawing. The difference is that with their right hand they indicate not only the position, but also if the hand is pointing or not (with a pinch gesture) while in our system the right hand indicates only the position, which is cognitively simpler for users: one hand used for pointing, the other for commands.

\section{Design of Gestures}

During this research we developed about 20 gestures classified into two groups of gestures, although most of the gestures are improvements of others. The first group of gestures is called technological and is designed to ensure robust and fast gesture recognition. The second gesture group, called iconic, was built to be ergonomic and easier to memorize by users. Two different sets of gestures were finally chosen from the 20 gestures and compared through a user evaluation. For both vocabularies the left hand is used for the commands and the right hand for the pointing (see Fig. 1).

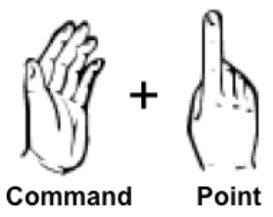

Fig. 1. Point + Command two handed gestural interaction paradigm 
For the finger detection the Candescent NUI library ${ }^{2}$ was used. This library gives the number of recognized fingers per hand as well as their location (fingertip), the volume of the hand, the palm position etc. For the pointing, which is done with the right hand, the highest point is used. Due to this fact, the hand can have any desired posture, which helps to reduce fatigue, although for the user it is easier to have only one finger outstretched. This prevents the cursor from switching from one finger to another if the user makes small rotations of the hand while he or she moves the cursor. In both gesture vocabularies the rotations and the zooms are continuous and thus clutch free. This means that the hand does not have to be repositioned during the gesture.

The rotation and the zoom are implemented in such a way that the center of the rotation and the focus point for zooming are always the middle point of the figure (marked with a blue dot, see Fig. 6).

\subsection{Technological Gesture Vocabulary}

The first gesture vocabulary is called technological and is designed to facilitate the efficiency and reliability of the gesture recognition. For this gesture set, sliding movements are used for the rotation and zoom. In order to activate the two actions, only one finger has to be present (see Fig. 2). As soon as only one finger is detected by the system, a red square appears on the screen with arrows and the names of the actions (Fig. 2). It serves as a neutral zone to prevent undesired action in the system. To execute an action (rotation or zoom) the finger has to go outside the red square. The two actions, rotation and zoom, cannot be done together.
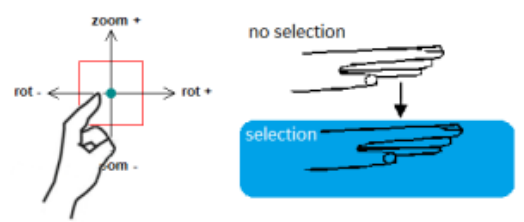

Fig. 2. The technological gesture set; on the left side rotation and zoom and on the right side selection

The user has to move the finger horizontally to rotate an object (see Fig. 3-b); left to rotate left, and right to rotate right. To perform a zoom the user has to move the finger vertically (see Fig. 3-c); up to zoom in and down to zoom out. The selection is done by showing the entire hand and making a vertical movement downwards, if the hand is below a certain threshold, shown with a blue rectangle (see Fig. 3-d), a selection is performed and if the hand moves up, a release is performed.

This gesture vocabulary requires only two postures (showing one finger and showing all fingers) for the left hand which supports robust gesture recognition and due to the small number of postures, it is also easy to remember for the user.

${ }^{2}$ http: //candescentnui.codeplex.com/ 

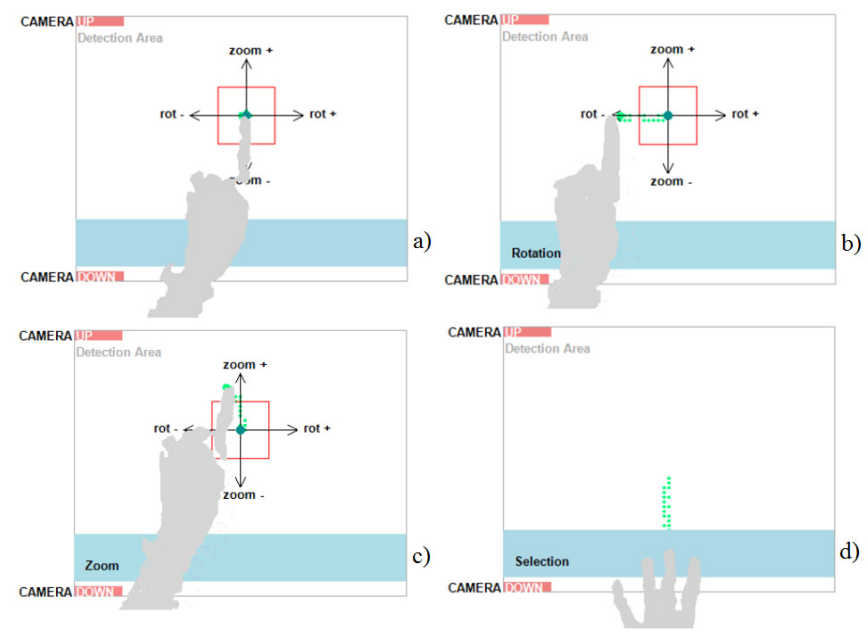

Fig. 3. Feedback technological gesture vocabulary

\subsection{Iconic Gesture Vocabulary}

For the second gesture set we followed a different approach than we did for the first gesture set and designed the gestures to be more natural and easier to memorize by users. In this iconic gesture vocabulary the rotation and the zoom are operated with a circular movement (see Fig. 4). For doing a rotation, only one finger has to be outstretched. For a zoom, two fingers must be outstretched. As feedback, a line is drawn between the activation point (darker green in Figure 5) and the current point (brighter green in Figure 5).

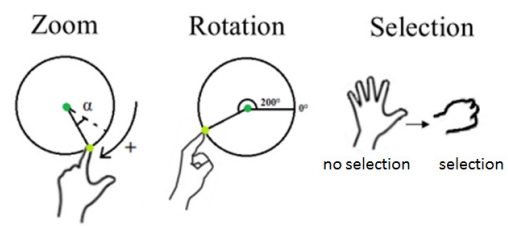

Fig. 4. The iconic gesture set; on the left side the zoom gesture, in the middle the rotation gesture and on the right side the selection gesture

The rotation gesture contains four parts. The first part is for the activation of the gesture, which is done by hiding all but one finger. At this point a dark green point is shown in the feedback (see dark green point in Figure 5 a). In the second part the user has to move the hand in one direction. Now, a violet line is drawn in the feedback (see Figure 5 a). As a third step the user has to move his hand around the green middle point. To stop the gesture, which is the fourth step, the user has to stretch out all fingers. The angle between the activation and the end is the angle which is used to rotate the object. In fact the object is already turning while the gesture is performed. If the user moves with a small radius around the center point (initial point), the rotation 
is fast but the user has less precision. Otherwise, if the user moves his hand further outside the center, the object turns more slowly and the user thus has more precision.

The difference between the zoom and the rotation gestures is the posture, which the user has to make in order to activate the gesture. For the zoom gesture, the user has to have two fingers outstretched (see Figure $5 \mathrm{~b}$ ). The angle of this technique obviously cannot be mapped directly to the zoom level. Therefore we compare the actual angle to the angle before and then do either a small zoom in or zoom out.

The selection in this gesture vocabulary works like grabbing an object with the hand. For a selection the user has to make a fist (see Figure 5-c) and for a release the user has to reopen the hand again.
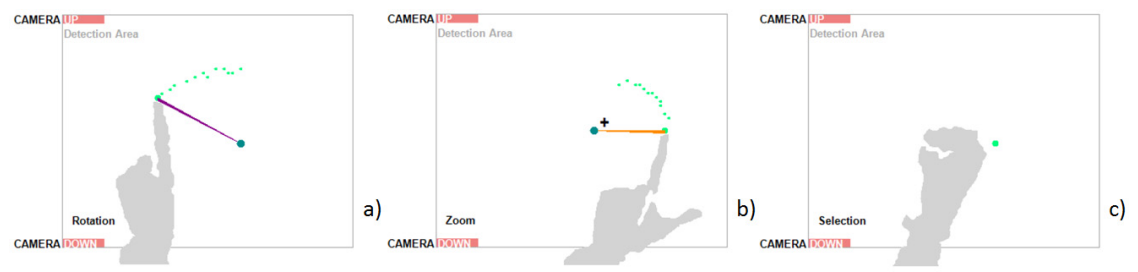

Fig. 5. Feedback iconic gesture vocabulary

\section{Test Application}

The developed evaluation application permits to evaluate the four commands (se-lection, drag \& drop, rotate and zoom) first separately and then together (see Fig. 6). Before the five exercises (the four commands separately and then together) each user has to do a training session to make sure that they have fully understood how to perform the gestures (see Fig. 6-a). The training takes a minimum of 90 seconds but can be longer if the user needs more training. Before each task there is a small animation displayed on the screen in which the task is shown to the user.

In the first level, which is the selection level (see Fig. 6-b), the user has to click on round targets. There are 12 round targets arranged on a circle. As soon as the first circle is clicked the $2^{\text {nd }}$ will be highlighted and so on, as proposed in part 9 of the ISO 9241 standard for non-keyboard input devices (multi-directional tapping test). In the second level, which is the drag \& drop level, the user has to move a circle into a zone. In Fig. 6-c the orange circle has to be moved into the blue rectangle. The task is validated when the blue middle point of the circle is within the rectangle. In the rotation level, which is shown in Fig. 6-d, several geometrical objects have to be rotated in order to fit the target which is represented by a border. Since the object only has to be rotated, the "figure to rotate" and the final target have the same center point. In order to make the task possible to accomplish, a small tolerance is allowed and the target turns green when the task is accomplished. The resizing level is shown in Fig. 6-e. This level acts very similarly to the rotation level. The final target is shown by the border of the figure and the center points are the same. Also for this level, there is a small tolerance which is allowed and the target turns green when the task is 
accomplished. The final level is shown in Fig. 6-f. This final level combines the selection, drag \& drop, rotation and resizing. The user has to select a geometrical object, move it to the right place, turn it to the correct angle and zoom it to the correct size. These actions can be done in any desired order.

To configure the application an xml file is used. The file contains all the pieces with their location, size and angle. Further it is possible to configure whether the piece can be zoomed, rotated, selected and moved. In the configuration file, the final location, size and orientation of pieces (used for the levels rotation, resize and final) are also specified.

While doing the different commands with the left hand, the right hand is used for pointing. As pointer feedback we use a cross-hair feedback i.e. small red circle with a cross (see Fig. 6 a-f). The selection command evaluation is based on part 9 of the ISO 9241 standard for non-keyboard input devices and Fitt's law (multi-directional tapping test).

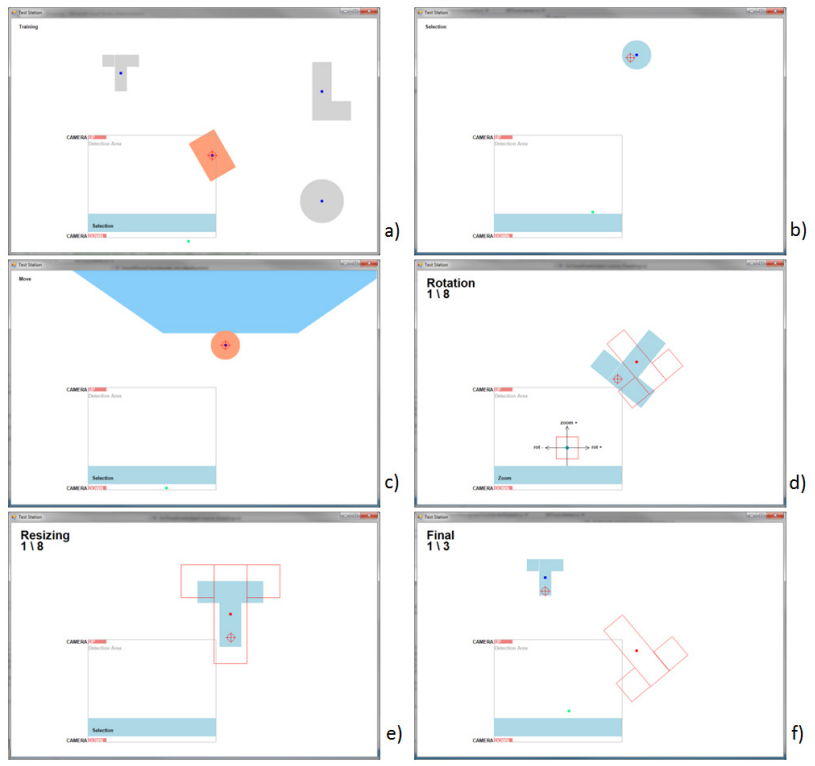

Fig. 6. The test application: the training panel (a) and the 5 exercises (b-f)

The objects in the test application can either be gray (normal state), blue (cursor is over it) or orange (selected). For the target objects, only the border is shown in red. If an object reaches the target, it becomes green and a message is displayed (see Fig. 7).

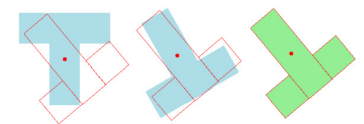

Fig. 7. Feedback geometrical objects 


\section{$5 \quad$ Evaluation and Results}

We conducted a within subject user evaluation with 10 users to compare the two gesture vocabularies. The comparison of the gesture vocabularies was done by comparing the time needed by the users to accomplish the tasks. In our evaluation all the users tested both gesture vocabularies. The gesture vocabularies were counter balanced to reduce carry over effects (fatigue and learning). For each gesture vocabulary the users had to select 12 objects as well as to move 12 objects. Further, the user had to rotate 8 objects and resize 8 objects. In the final level, the users had to move, rotate and resize 3 objects. The evaluation was done on a personal computer with an office chair with arm rests. Several paired samples t-tests were conducted. Significant difference was found between the two rotation gestures: $\mathrm{t}(9)=3.72, \mathrm{p}=.0048$. Significant difference was also found between the two resize gestures: $t(9)=2.57, p=.03$. For the other pairs there was no significant difference. The results are presented in Table 1.

Table 1. Results of the user evaluation

\begin{tabular}{|c|c|c|c|c|c|c|c|c|c|c|}
\hline & & mean & median & low & high & $\begin{array}{r}\text { std } \\
\text { dev }\end{array}$ & $\begin{array}{r}t- \\
\text { value }\end{array}$ & $\mathrm{df}$ & t-test & \\
\hline \multirow{2}{*}{ Selection } & techno. & 4.621 & 4.425 & 2.95 & 6.99 & 1.32 & \multirow{2}{*}{0.737} & \multirow{2}{*}{9} & \multirow{2}{*}{0.48} & \multirow{2}{*}{---- } \\
\hline & iconic & 4.26 & 4.184 & 3.32 & 6.52 & 0.915 & & & & \\
\hline \multirow{2}{*}{ Move } & techno. & 5.475 & 4.961 & 3.83 & 8.91 & 1.44 & \multirow{2}{*}{1.118} & \multirow{2}{*}{9} & \multirow{2}{*}{0.266} & \multirow{2}{*}{---- } \\
\hline & iconic & 4.87 & 4.852 & 3.92 & 6.97 & 0.89 & & & & \\
\hline \multirow{2}{*}{ Rotation } & techno. & 17.57 & 16.18 & 9.14 & 28.7 & 5.69 & \multirow{2}{*}{3.72} & \multirow{2}{*}{9} & \multirow{2}{*}{0.0048} & \multirow{2}{*}{$* *$} \\
\hline & iconic & 14.07 & 14.07 & 9.18 & 22.1 & 4.13 & & & & \\
\hline \multirow{2}{*}{ Resizing } & techno. & 14.63 & 14.61 & 9.61 & 19.8 & 2.79 & \multirow{2}{*}{2.57} & \multirow{2}{*}{9} & \multirow{2}{*}{0.03} & \multirow{2}{*}{$*$} \\
\hline & iconic & 12.28 & 12.06 & 9.16 & 15.7 & 1.86 & & & & \\
\hline \multirow{2}{*}{ Final } & techno. & 54.3 & 54.74 & 32.5 & 69.1 & 12.3 & \multirow{2}{*}{-0.25} & \multirow[t]{2}{*}{9} & \multirow{2}{*}{0.808} & \multirow{2}{*}{---- } \\
\hline & iconic & 55.34 & 56.96 & 31.8 & 73.3 & 12.1 & & & & \\
\hline
\end{tabular}

null hypothesis significance level: $*=<0.05 ; * *=<0.01 ; * * *=<0.001 ; *=<0.05$

After the first gesture set, as well as after the second gesture set, the users had to fill out a questionnaire, based on the one proposed in ISO 9241 part 9 annex C. The questions have a Likert scale from 1 to 7 , where 7 is the best. For the fatigue questions it is the inverse, i.e. 1 is the best. This questionnaire permitted to measure user satisfaction as well as fatigue. The results of the questionnaire are presented in Table 2 .

Table 2. Results of the questionnaire based on the ISO standard

\begin{tabular}{|c|c|c|c|c|c|c|c|c|c|c|c|c|c|c|c|c|}
\hline & 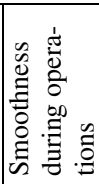 & 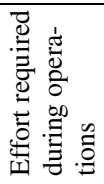 & 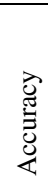 & 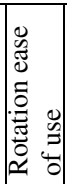 & 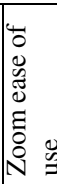 & 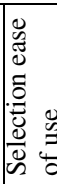 & 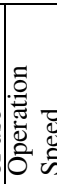 & 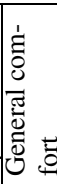 & 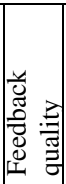 & 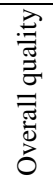 & 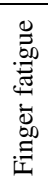 & 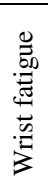 & 窇 & 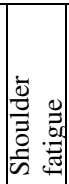 & 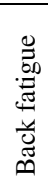 & 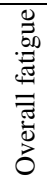 \\
\hline Techno & 5.4 & 2.5 & 4.4 & 4.2 & 4.7 & 4.4 & 5.1 & 5.0 & 5.9 & 5.2 & 1.8 & 1.3 & 5.4 & 2.5 & 1.4 & 3.8 \\
\hline Iconic & 5.1 & 3.0 & 5.2 & 4.3 & 4.9 & 6.5 & 5.2 & 4.4 & 5.1 & 5.4 & 2.3 & 1.3 & 5.3 & 2.6 & 1.5 & 4.0 \\
\hline
\end{tabular}


To analyze the perceived quality of the two gesture vocabularies we conducted Wilcoxon signed rank tests, since the data is not normally distributed. We found only two significant differences for "Selection ease of use" and "feedback quality". In general the commands are slightly better rated for the iconic gesture vocabulary but only the selection is significantly better. For the fatigue the technological gesture set is rated slightly better but not significantly. The general comfort seems to be better for the technological vocabulary, although not significantly. This could be influenced by the fatigue. The second significant difference is the feedback which was better rated for the technological gesture vocabulary.

There is a reason why the fatigue was rated worse for the iconic gestures, namely that the users have to switch the commands for zooming and rotating in the iconic gestures set (two different activations; one and two fingers) whereas for the technological gesture set the activation gesture (one finger) is the same for both and thus it is faster to change between those gestures.

\section{Conclusion}

In this article we presented two gestural vocabularies with a 2 handed point \& command paradigm. The first one is based on a technological approach which is easier to detect. The second one is based on an iconic approach and is therefore more natural to use. Secondly, the paper presented a test application on which the gestures were compared. Finally a user evaluation was presented.

By analyzing the results of user performance in the user evaluation we found significant differences for the rotation and the resize gestures. Overall, the iconic gesture vocabulary had slightly better performance. Concerning the user perception, users preferred the selection of the iconic gesture vocabulary, which is more natural (grabbing an object), over the more technological one. For the other commands there was no significant difference. In this user evaluation we did not have the opportunity to check how easily the gestures were remembered. Analyzing the questionnaire and the comments of the users during the evaluation, we suppose that the iconic gesture set is easier to remember. For those reasons in future work we suggest to develop more gestures which are more natural to perform than gestures which are easy to recognize, in order to augment the acceptance of users as well as the easiness of performing the gestures.

We plan to develop other gestures which would be more subtle but still easy to perform and easy to remember by users, but which would require machine learning because they would be too subtle for stable recognition otherwise. In a second step we would then compare the machine learning gestures with the ones proposed in this paper. For this comparison we would like to make two evaluations, the first one with the application proposed in this paper and the second one with another application such as Google Earth. In the future we would also like to compare two handed gestures with the paradigm where one hand is used for pointing and one hand for the gesture command, versus one handed gestures, since then both hands could do 
gestures at the same time. We also plan to design gestures in combination with speech input and would like to compare those with the two handed gesture paradigm presented in this paper.

Acknowledgment. Grateful acknowledgement for proofreading and correcting the English goes to Agnes Lisowska Masson.

\section{References}

1. Nancel, M., Wagner, J., Pietriga, E., Chapuis, O., Mackay, W.: Mid-air pan-and-zoom on wall-sized displays. In: Proceedings of the 2011 Annual Conference on Human Factors in Computing Systems, CHI 2011, p. 177. ACM Press, New York (2011)

2. Gallo, L., Placitelli, A., Ciampi, M.: Controller-free exploration of medical image data: experiencing the Kinect. Computer-Based Medical (2011)

3. Song, P., Goh, W.B., Hutama, W., Fu, C.-W., Liu, X.: A handle bar metaphor for virtual object manipulation with mid-air interaction. In: Proceedings of the 2012 ACM Annual Conference on Human Factors in Computing Systems - CHI 2012, p. 1297 (2012)

4. Zigelbaum, J., Browning, A., Leithinger, D., Bau, O., Ishii, H.: g-stalt: a chirocentric, spatiotemporal, and telekinetic gestural interface. In: Proceedings of the Fourth International Conference on Tangible, Embedded, and Embodied Interaction - TEI 2010, p. 261. ACM Press, New York (2010)

5. Mistry, P., Maes, P., Chang, L.: WUW - Wear Ur World - A Wearable Gestural Interface. In: Proceedings of the 27th International Conference Extended Abstracts on Human Factors in Computing Systems - CHI EA 2009, p. 4111. ACM Press, New York (2009)

6. Boulos, M.N.K., Blanchard, B.J., Walker, C., Montero, J., Tripathy, A., Gutierrez-Osuna, R.: Web GIS in practice X: a Microsoft Kinect natural user interface for Google Earth navigation. International Journal of Health Geographics 10, 45 (2011)

7. Stellmach, S., Jüttner, M., Nywelt, C., Schneider, J., Dachselt, R.: Investigating Freehand Pan and Zoom. In: Reiterer, H., Deussen, O. (eds.) Mensch \& Computer 2012: Interaktiv Informiert - Allgegenwärtig und Allumfassend!?, pp. 303-312. Oldenbourg Verlag, München (2012)

8. Gustafson, S., Bierwirth, D., Baudisch, P.: Imaginary Interfaces: Spatial Interaction with Empty Hands and without Visual Feedback. In: Proceedings of the 23nd Annual ACM Symposium on User Interface Software and Technology - UIST 2010, p. 3. ACM Press, New York (2010) 